\title{
An Agent-Based Approach for Manufacturing Enterprise Integration and Supply Chain Management
}

\author{
W. Shen and D. H. Norrie \\ Div. of Manufacturing Engineering, The University of Calgary \\ 2500 University Dr. NW, Calgary, AB, Canada T2N $1 N 4$ \\ Tel: (403) 220-4165 Fax: (403) 282-8406 \\ E-mail: [wshen | norrie]@enme.ucalgary.ca \\ URL: http://imsg.enme.ucalgary.cal
}

\begin{abstract}
Improving supply chain management is very important for increasing competitive position and profitability. Manufacturing enterprises are now moving towards open architectures for integrating their activities with those of their suppliers, customers and partners within wide supply chain networks. This paper presents an agentbased approach for manufacturing enterprise integration and supply chain management to meet such requirements. A hybrid agent-based architecture is proposed, the main features of such an architecture are then described, and a prototype implementation is presented.
\end{abstract}

\section{Keywords}

Enterprise integration, distributed manufacturing systems, supply chain management, agent, multi-agent systems, mediator

\section{INTRODUCTION}

Manufacturing enterprises are confronted with growing competition, the evolution of new markets and increasingly complex global political and economic scenarios. The need for cheaper and more cost-effective products is evident. Improving supply chain management is a key mechanism for increasing competitive position and profitability. Consequently, enterprises are moving towards open architectures for integrating their activities with those of their suppliers, customers and partners 
within wide supply chain networks. Agent-based technology provides a natural way to design and implement such manufacturing environments.

Recently, a number of architectures for manufacturing enterprise integration and supply chain management have been proposed. Pan and Tenenbaum (1991) described a software Intelligent Agent (IA) framework for integrating people and computer systems in large, geographically dispersed manufacturing enterprises. Peng et al (1998) presented a real-word application of a multi-agent system to enterprise integration for manufacturing planning and execution. Roboam and Fox (1992) proposed an Enterprise Management Network (EMN) to support the integration of activities of the manufacturing enterprise throughout the production life cycle with six levels: Network Layer, Data Layer, Information Layer, Organization Layer, Coordination Layer and Market Layer. Barbuceanu and Fox (1997) further described a supply chain network architecture using autonomous agents with a specially developed coordination language. The AIMS (Agile Infrastructure for Manufacturing Systems) team proposed to create an open, scalable infrastructure for agile manufacturing, and to demonstrate its effectiveness in pilot production (Park et al 1993). The infrastructure was intended to provide standardized ways of accessing a wide variety of agile production services over local network as well as the Internet. AARIA (Autonomous Agents for Rock Island Arsenal) (Parunak et al 1997) is an industrial-strength, agent-based factory scheduling and simulation system developed for a defense manufacturing facility. Fischer (1994) proposed a hierarchical planning structure consisting of six layers: the layer of the production planning and control system, the layer of the shop floor control system, the task coordination layer, the task planning layer, the task execution layer and the machine control layer. MADEFAST (Cutkosky et al 1996) was a DARPA DSO-sponsored project to demonstrate technologies developed under the MADE (Manufacturing Automation and Design Engineering) program.

The rest of this paper is organized as follows: Section 2 reviews some of our previous research work; Section 3 presents an agent-based approach for manufacturing enterprise integration and supply chain management; Section 4 describes a prototype implementation; Section 5 gives concluding remarks and perspectives.

\section{PREVIOUS RESEACH WORK}

The research work presented in this paper is based on our earlier research projects: MetaMorph I (Maturana and Norrie 1996), ABCDE (Agent-Based Concurrent Design Environment) (Balasubramanian et al 1996), and DIDE (Distributed Intelligent Design Environment) (Shen and Barthès 1995). In this section, we give a brief overview of these research projects.

\subsection{The MetaMorph I and ABCED projects}

MetaMorph I was developed at The University of Calgary using the mediatorcentric federation architecture for intelligent manufacturing (Maturana and Norrie 1996). In this particular type of federation architecture, agents can link with 
mediators to find other agents in the environment. Additionally, mediators assume the role of system coordinators by promoting cooperation among agents and learning from the agents' behavior. These mediators are similar to facilitators in PACT (Cutkosky et al 1993), but with additional functionality such as coordination and learning. In MetaMorph I, agents are used to represent manufacturing devices and products or parts to be fabricated, while mediators are used to coordinate the interactions among agents. The architecture has been named MetaMorphic, since a primary characteristic is its changing form, structure, and activity as it dynamically adapts to emerging tasks and changing environment.

In MetaMorph I, the dynamic mediator structure is a distributed decision-making support system for coordinating the activities of a multi-agent system. This coordination involves three main phases: (1) subtasking; (2) creation of virtual communities of agents (coordination clusters); and (3) execution of the processes imposed by the tasks. These phases are developed within the coordination clusters by distributed mediators together with other agents representing the physical devices. The coordination clusters are initialized through mediators, which can dynamically find and incorporate those other agents that can contribute to the task. ABCDE (Balasubramanian et al 1996) was developed in the same research group. It was integrated with the MetaMorph I system through a design mediator. The $\mathrm{ABCDE}$ architecture includes an environment manager, feature agents, part agents, and $C A D$ physical layers. $A B C D E$ agents interact with the shop-floor mediators to obtain manufacturability assessments during the product design process.

\subsection{The DIDE project}

The DIDE (Distributed Intelligent Design Environment) was developed at the University of Technology of Compiègne by the first author using the autonomous agent approach, originally for engineering design, but its general architecture can also be used to develop advanced distributed manufacturing systems or distributed integrated $\mathrm{CAD} / \mathrm{CAM}$ systems. Its objective was to integrate existing engineering tools, like CAD/CAM tools, database systems, or knowledge-based systems, into a truly open system, that is, a system for which users can freely add or remove agents without having to halt or to reinitialize the work in progress.

The general architecture of DIDE is organized as a population of asynchronous cognitive agents for integrating engineering tools and human specialists in an open environment. Each tool (or interface for human specialist) can be encapsulated as an agent. Engineering tools and human specialists are connected by a local network and communicate via this network. Each of them can also communicate directly with any other agent located in other local networks using the Internet. All agents are independent and autonomous. They exchange design data and knowledge via a local network or the Internet. There is no facilitator structure as in PACT (Cutkosky et al 1993), nor mediator structure as in MetaMorph I (Maturana and Norrie 1996). There is no any static global control structure in the system.

The communication among DIDE agents used the OSACA protocol (Scalabrin 1996) with five types of messages: Request, Inform, Notice, Announce and Bid. 


\section{AN AGENT-BASED APPROACH FOR ENTERPRISE INTEGRATION AND SUPPLY CHAIN MANAGEMENT}

\subsection{A hybrid architecture - MetaMorph II architecture}

The MetaMorph II project began at the beginning of 1997. Its objective is to integrate the manufacturing enterprise's activities such as design, planning, scheduling, simulation, execution, and product distribution, with those of its suppliers, customers and partners into an open, distributed intelligent environment. For this purpose, we propose a hybrid agent-based architecture combining and extending the architectures used in our previous projects MetaMorph I (Maturana and Norrie 1996), ABCDE (Balasubramanian et al 1996) and DIDE (Shen and Barthès 1995).

In this hybrid architecture, the system is primarily organized at the highest level through 'subsystem' mediators which are connected via the Internet/Intranet (see Figure 1). Each subsystem is connected (integrated) to the system through a special mediator. Each manufacturing enterprise must have at least one Enterprise Mediator. It can be considered as the administration center of the enterprise. All other mediators should register with it. Each subsystem itself can be an agentbased system (e.g., agent-based manufacturing scheduling system), or any other type of systems like feature-based design system, knowledge-based material management system, and so on. Agents in a subsystem may also be autonomous agents at the subsystem level. Some of these agents may also be able to communicate directly with other subsystems or the agents in other subsystems.

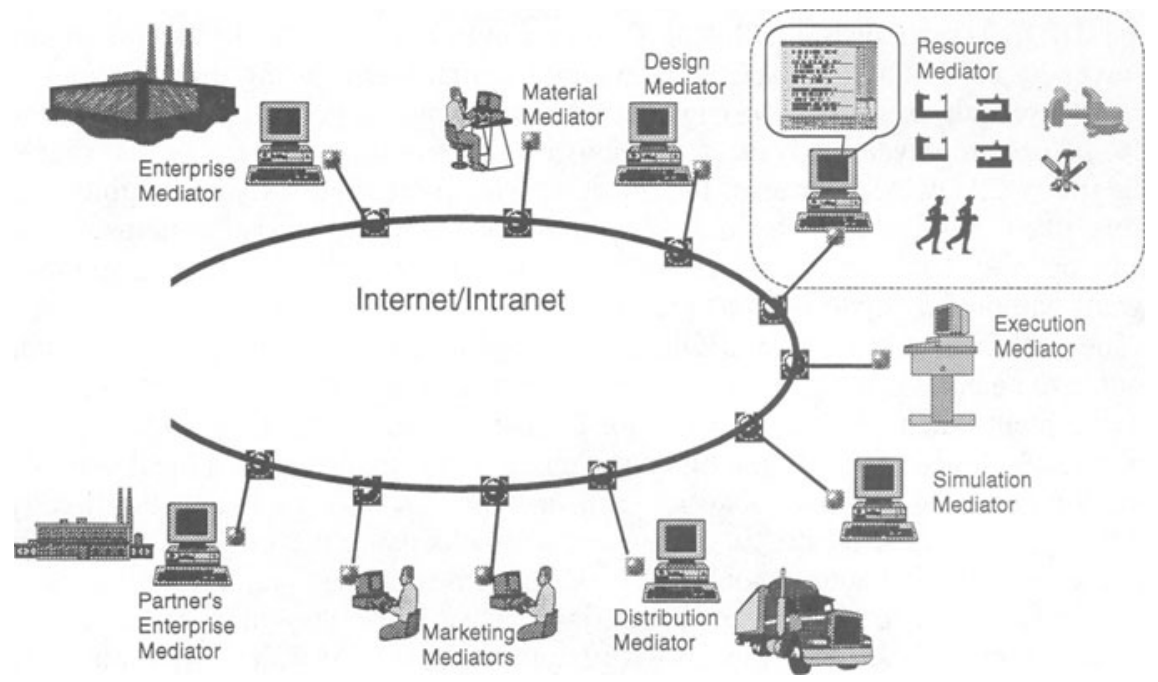

Figure 1. Mediators Connected via the Internet/Intranet. 
Figure 2 shows the functional architecture of MetaMorph II from the point of view of supply chain management. In this approach, partners, suppliers and customers are dynamically connected with the main manufacturing enterprise through mediators (partners' enterprise mediators, suppliers' mediators and marketing mediator) by a supply chain network (via the Internet/Intranet). Such architecture may:

- increase the responsiveness of the enterprise to the market requirements;

involve customers in total supply chain optimization;

- achieve supply chain optimization through effective resource allocation;

- achieve dynamic optimization of materials management and inventory management;

realize total supply chain optimization including all linked enterprises;

- $\quad$ increase the effectiveness of information exchange and feedback.

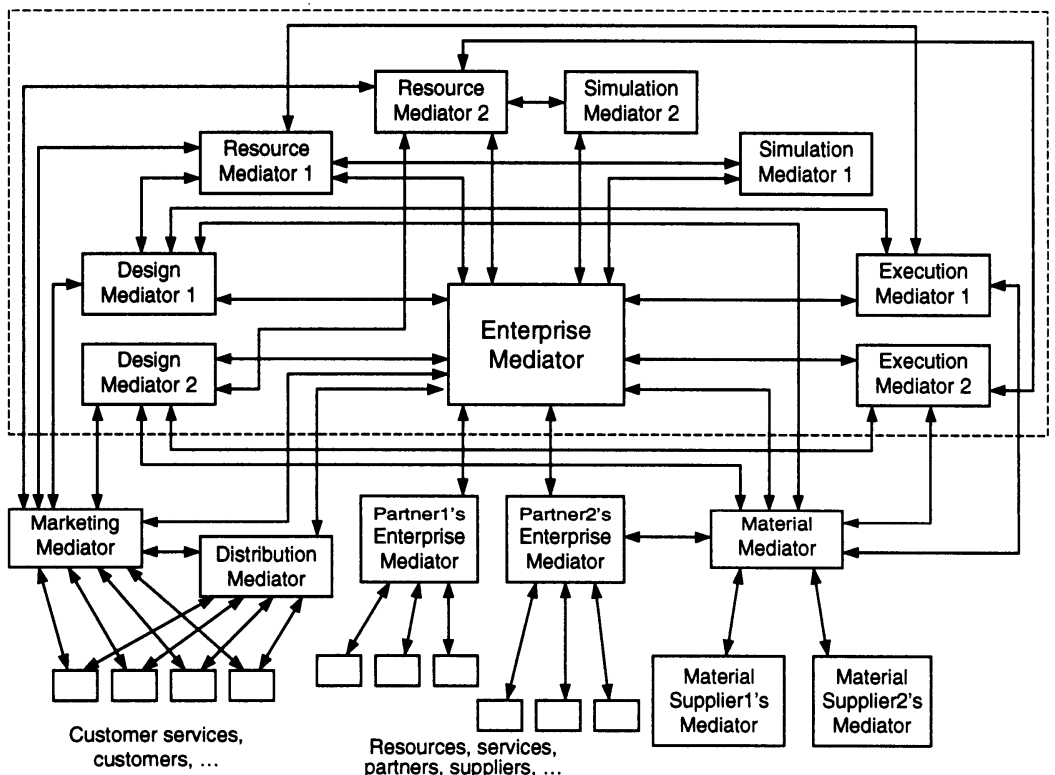

Figure 2. Functional Architecture of MetaMorph II

\subsection{Main features of the MetaMorph II architecture}

\section{Resource agents and mediator agents}

All manufacturing resources (e.g., machines. Tools, workers, AGVs, etc.) are modeled as resource agents. Resource agents are coordinated by layered mediator agents (also called mediators). For example, as shown in Figure 3, a Machine 
Mediator is used to coordinate all the machines in the shop floor; a Tool Mediator is used to coordinate all tools, and so on; and a high level Resource Mediator is used to coordinate low-level mediators, like Machine Mediators, Tool Mediators, Worker Mediators, Transportation Mediators, etc. In this type of architecture, the system level organization of mediators may appear to be hierarchical, but there is neither hierarchical control structure nor hierarchical coordination mechanism. A machine agent can also communicate and negotiate directly with a Worker Mediator, worker agents, a Tool Mediator and tool agents, and so on. In other words, the communication between two different types of resource agents (e.g., between a machine agent and a worker agent) can be direct, rather than always through facilitators or mediators as in the architectures proposed by other researchers (Cutkosky et al 1993; Wiederhold 1992).

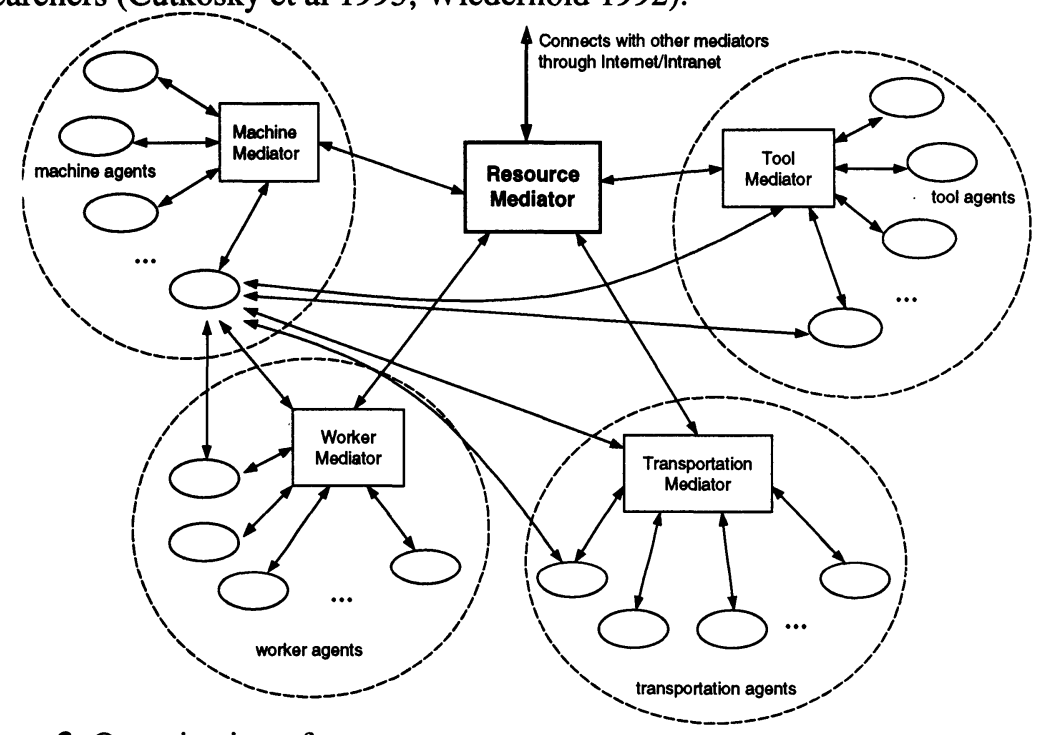

Figure 3. Organization of resource agents.

\section{Hybrid architecture}

The hybrid characteristic of the proposed architecture can be found at two levels: the system level and the subsystem level.

As mentioned in Section 3.1, at the system level, each subsystem can be an agent-based system or any other types of systems. It is therefore possible to integrate existing different tools (software or hardware) into the system, so as to resolve the legacy problems.

At the subsystem level, if the subsystem is an agent-based system like shop floor resource scheduling system, each resource agent may communicate or negotiate directly with any other agents in the subsystem (as described previously) or with any other subsystems. 


\section{Open and heterogeneous environment}

Because of its modular architecture, the system developed using such architecture is a true open environment, i.e., one can dynamically integrate new subsystems to or remove existing subsystems from the system without stopping and reinitializing the working environment.

The implementation environment of such architecture can be heterogeneous due to its TCP/IP or other type of appropriate communication.

\section{Design and manufacturability assessments}

Several design subsystems can be connected to the distributed manufacturing system simultaneously. Such design subsystems may be either agent-based intelligent design systems like DIDE and ABCDE, or other types of design systems like feature-based design systems, knowledge-based multi-expert design systems. Each design subsystem connects to the manufacturing system through a Design Mediator which serves as the coordinator of this subsystem and its only interface to the whole system.

Geometric and functional specifications, availability of raw materials, and the capability and availability of shop-floor resources each have a major influence on manufacturability. A design may be manufacturable under one combination of product requirements and shop-floor resources, but not under another. The proposed architecture can provide an immediate manufacturability assessments as was the case in MetaMorph I. As a product part is progressively designed by repeated instantiation of features, manufacturability is evaluated by resource agents for every instantiation. Design Mediators and Resource Mediators ensure the coordination among design parts and resource agents. Design subsystems (or design agents) interact with resource agents via Resource Mediators to obtain manufacturability assessments during the product design process. This process not only ensures the manufacturability of a product, but also results in incremental identification of general process plans.

\section{Integration of planning and scheduling}

Traditional approaches to planning and scheduling do not consider the constraints of both domains simultaneously. In spite of being sub-optimal, these approaches have been in vogue due to the unavailability of a unified framework. The proposed architecture provides a possible way to integrate planning and scheduling activities through manufacturing enterprise level coordination between Design Mediators and Resource Mediators which in turn coordinate resource agents at the shop floor level. Experimental work carried out during the MetaMorph I project demonstrated such integration (Maturana et al 1996).

\section{System simulation and execution control}

Simulation Mediators will be developed to carry out production simulation and forecasting. Each Simulation Mediator corresponds to one Resource Mediator and therefore to one shop floor.

Execution Mediators will also be developed to coordinate the execution of the machines, AGVs, and workers as necessary. By such approach, manufacturing 
devices (e.g., machines) can be connected directly to the manufacturing system. Each shop floor is, in general, assigned with one Execution Mediator. Execution Mediators can be considered as the manufacturing system's interfaces with related hardware. This area relates to the intelligent control project in our research group (Brennan et al 1997).

\section{Customer involvement in supply chain network}

This will be realized by easy-to-use interfaces for marketing engineers and end customers to request product information (performance, price, manufacturing period, etc), select a product, request modifications to a particular specification of a product, and send feedback to the enterprise. By this approach, the customer's requirements can be directly incorporated within total supply chain optimization.

\section{Interface agents for human involvement}

As in DIDE, special graphic interfaces need to be developed to allow the participation of the human specialists or end users. For example, at the Marketing Mediator level, a 3D graphic interface is being developed on 3D Studio ${ }^{\mathrm{TM}}$ for allowing customers to view and select products. The Enterprise Mediator similarly needs an interface for the enterprise manager, the Material Mediator needs an interface for the material supply manager, and the Resource Mediator needs an interface for the production manager.

In general, human beings would not have direct control over other mediators and agents in this system. What the human specialists may do is to start and stop the system, to monitor or obtain desired information, to respond to the requests of the system for decision-making or conflict resolution, and to reconfigure the system via special interfaces.

\section{Integration with partners' activities}

Manufacturing enterprises need always to collaborate with their partners for parts fabrication, raw materials supply etc. In this architecture, each partner has an Enterprise Mediator (see Figures 1 \& 2). Each partner's Enterprise Mediator registers with the Enterprise Mediator of the principal manufacturing enterprise as do other subsystem mediators. A subsystem mediator may also cooperate directly with other Enterprise Mediators, e.g., a Material Mediator may communicate directly with the Enterprise Mediator of a raw material supply company.

\section{Integration of material supply and management}

A Material Mediator will be developed to coordinate a special subsystem for material supply and management.

\section{Integration of product distribution management}

A Distribution Mediator will be developed to integrate some software for product distribution management. 


\subsection{Internal structure of a mediator}

Mediators are also agents, called mediator agents. They are different from facilitators as in PACT (Cutkosky et al 1993). The main difference between a mediator and a facilitator is that a facilitator provides primarily message services, but a mediator assumes additionally the role of system coordinator by promoting cooperation among intelligent agents and learning from the agents' behavior.

For carrying out the above functionality, a mediator should be composed of following components (Figure 4):

- a network interface for connecting it to the system

- a communication interface for incoming and outgoing messages

- knowledge about itself

- detailed knowledge about the agents coordinated by itself

- knowledge about other mediators

- knowledge about its environment

- knowledge about the working projects or products to be manufactured

- reasoning mechanisms for using its knowledge

- learning mechanisms for updating its knowledge

- control mechanisms for controlling its actions and events

A mediator is an agent, and a resource agent is also an agent. So they have similar architecture, but a resource agent does not need detailed knowledge about other agents and mediators, about its environment, about the whole project or parts to be manufactured, and so on.

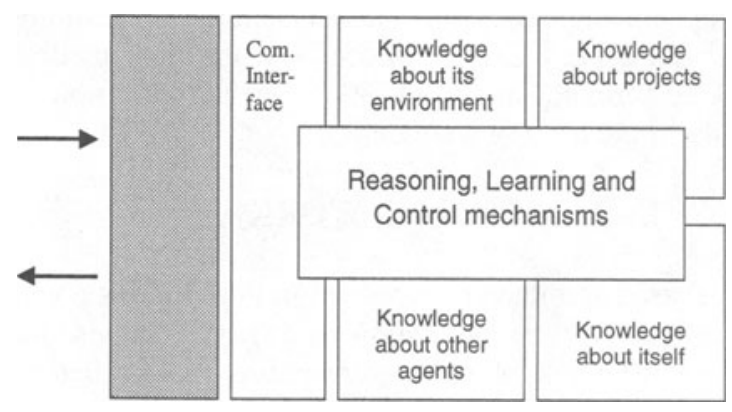

Figure 4. Internal structure of a mediator.

\section{PROTOTYPE IMPLEMENTATION}

The current implementation of the proposed architecture consists of four mediators: Enterprise Mediator, Design Mediator, Resource Mediator and Marketing Mediator.

The Enterprise Mediator can be considered as the administration center of the manufacturing enterprise. Other mediators register with this mediator. The Design Mediator is being used to integrate a feature-based intelligent design system. The functions of the design module include: (1) generating design candidates from the design functional requirements; (2) modeling design geometry; and (3) 
representing the design using manufacturing features. The Resource Mediator is used to coordinate an agent-based dynamic manufacturing scheduling subsystem (see Shen and Norrie 1998 for details). In the present prototype implementation, at the resource level, only machine agents and worker agents are so far implemented. The Marketing Mediator is used to integrate the customer services (marketing subsystem) into the system. The functions of the marketing subsystem include: (1) using its class libraries (product catalogue) to show different product models on a 3D graphical interface according to the customers' requests; (2) if the requested product cannot be found in its libraries (product catalogue), sending design (functional) requirements to the Design Mediator for asking the design subsystem to design a new product; (3) requesting the product price for a special order with special product specifications or special due time.

In this implementation, all scheduling requests derive from a feature-based intelligent design system via a Design Mediator. Each product corresponding to a customer order has been decomposed into related manufacturing features. Each manufacturing feature can be realized by a manufacturing task (operation). These manufacturing tasks are organized in a graph data structure representing the sequence to produce the corresponding manufacturing features. Each product is modeled as a part agent containing the information about this product including the feature data, due time, and its plan which will be assigned with times and resources during the scheduling process.

The current prototype is developed on a network of PCs. The main development language is VisualWorks ${ }^{\mathrm{TM}}$ (Smalltalk). The graphical interface for customer services is developed using $3 \mathrm{D}$ studio ${ }^{\mathrm{TM}}$ and Visual $\mathrm{C}++^{\mathrm{TM}}$. Communication among mediators is realized using TCP/IP protocol and the inter-mediator messages are formatted in KQML format (Finin et al 1993). The current implementation is being developed initially in the form of a simulation.

\section{CONCLUSION AND FUTURE WORK}

The hybrid agent-based architecture proposed in this paper is a general architecture for manufacturing enterprise integration and supply chain management. The implementation environment of such architecture can be heterogeneous. In the present case, TCP/IP communication is being used, as it was for MetaMorph I on a network of HP workstations, SUN workstations and PCs. It should be noted that a real implementation in a particular manufacturing enterprise does not need all components of this architecture and some mechanisms may also be simplified according to the real situation of the enterprise.

The proposed architecture has some similarities to the supply chain network architecture proposed by Barbuceanu and Fox (1997). However, the agents in their architecture are primarily autonomous agents and there is additional emphasis on developing a coordination language. Our architecture is primarily mediator-centric, and the objective of this current research project is to implement a practical agentbased manufacturing system using existing tools where possible. This architecture also differs from the agile infrastructure proposed by the AIMS team (Park et al 
1993) in that AIMS is Internet based, while this is agent-based and mediatorcentric.

MetaMorph II is an ongoing project. A major short-term research goal is to realize a prototype integrating intelligent feature-based design and dynamic manufacturing scheduling, in simulation mode, and then to implement this approach in a real manufacturing enterprise. The long-term research goal is to develop new mechanisms, techniques and tools for manufacturing enterprise integration and supply chain networks.

\section{REFERENCES}

Balasubramanian, S., Maturana, F. and Norrie, D. (1996) Multi-agent planning and coordination for distributed concurrent engineering. International Journal of Cooperative Information Systems, 5(2-3), 153-179.

Barbuceanu, M. and Fox, M. (1997) Integrating Communicative Action, Conversations and Decision Theory to Coordinate Agents, in Proceedings of Autonomous Agents'97, Marina del Rey, CA.

Brennan, R.W., Balasubramanian, S., and Norrie, D.H. (1997) Dynamic Control Architecture for Advanced Manufacturing Systems, in Proceedings of International Conference on Intelligent Systems for Advanced Manufacturing, Pittsburgh, PA.

Cutkosky, M.R., Engelmore, R.S., Fikes, R.E., Genesereth, M.R., Gruber, T.R., Mark, W.S., Tenenbaum, J.M. and Weber, J.C. (1993) PACT: An Experiment in Integrating Concurrent Engineering Systems. IEEE Computer, 26(1), 28-37.

Cutkosky, M.R., Tenenbaum, J.M. and Glicksman J. (1996) Madefast: Collaborative Engineering over the Internet. Communication of the ACM, 39(9), 78-87.

Finin, T., Fritzon, R., McKay, D. and McEntire, R. (1993) KQML - A Language and Protocol for Knowledge and Information Exchange. Tech. Report, University of Maryland, Baltimore.

Fischer, K. (1994) The Design of an Intelligent Manufacturing System, in Proceedings of the 2nd International Working Conference on Cooperating Knowledge-based Systems (CKBS'94), Dake Centre, University of Keele, UK.

Maturana, F. and Norrie, D. (1996) Multi-Agent Mediator Architecture for Distributed manufacturing. Journal of Intelligent Manufacturing, 7, 257-270.

Maturana, F., Balasubramanian, S. and Norrie, D.H. (1996) A Multi-Agent Approach to Integrated Planning and Scheduling for Concurrent Engineering, in Proceedings of the International Conference on Concurrent Engineering: Research and Applications, Toronto, Canada.

Pan, J.Y.C. and Tenenbaum, M.J. (1991) An intelligent agent framework for enterprise integration. IEEE Transactions on Systems, Man, and Cybernetics, 21(6), 1391-1408

Park, H., Tenenbaum, J. and Dove, R. (1993) Agile Infrastructure for Manufacturing Systems (AIMS): A Vision for Transforming the US Manufacturing Base. Defense Manufacturing Conference. 
Parunak, H.V.D., Baker, A.D. and Clark, S.J. (1997) The AARIA Agent Architecture: An Example of Requirements-Driven Agent-Based System Design, in Proceedings of the First International Conference on Autonomous Agents, Marina del Rey, CA.

Peng, Y., Finin, T., Labrou, Y., Chu, B., Long, J., Tolone, W.J. and Boughannam, A. (1998) A Multi-Agent System for Enterprise Integration, in Proceedings of the Third International Conference on the Practical Application of Intelligent Agents and Multi-Agents, London, UK.

Roboam, M. and Fox, M.S. (1992) Enterprise Management Network Architecture, in Artificial Intelligence Applications in Manufacturing (eds. Famili, A., Nau, D.S. and Kim, S.H.), The AAAI Press.

Scalabrin, E. (1996) Conception et Réalisation d'environnement de développement de systèmes d'agents cognitifs. Thèse de Doctorat, CNRS UMR Heudiasyc, Université de Technologie de Compiègne, France.

Shen, W. and Barthès, J.P. (1995) DIDE: A Multi-Agent Environment for Engineering Design, in Proceedings of the First International Conference on Multi-Agent Systems, San Francisco, CA, The AAAI press/The MIT press.

Shen, W. and Norrie, D.H. (1998) An Agent-Based Approach for Dynamic Manufacturing Scheduling, in Proceedings of Autonomous Agents'98 Workshop on Agent-Based Manufacturing, Minneapolis/ St. Paul, MN.

Wiederhold, G. (1992) Mediators in the architecture of future information systems. IEEE Computer, 25(3), 38-49.

\section{BIOGRAPHY}

Weiming Shen currently works as Research Associate in the Division of Manufacturing Engineering at The University of Calgary. He obtained his Bachelor and Master in Mechanical Engineering from Northern Jiaotong University, China in 1983 and 1986, and his PhD in Computer Science from the University of Technology of Compiègne, France in 1996. He worked as Lecturer in the Department of Mechanical Engineering at Northern Jiaotong University from 1986 to 1992 and was appointed as the executive director of CAD/CAM Laboratory in 1989. His current research is focused on agent-based approaches for concurrent engineering design and intelligent manufacturing.

Douglas H. Norrie currently holds the Nortel Chair in Intelligent Manufacturing at The University of Calgary, Alberta, Canada. Formerly, he was Head of the Division of Manufacturing Engineering at The University of Calgary. He is also Professor of Mechanical Engineering and Adjunct Professor of Computer Science at the same institution. His research interests are in Intelligent Systems and, in particular, in Multi-Agent Applications in Manufacturing. 